\title{
Registro continuado por 24 horas del pH esofágico en el diagnóstico de reflujo gastroesofágico en niños
}

\author{
Patricio Varela B. '; Jorge Godoy L. ${ }^{1}$
}

\begin{abstract}
Resumen
El registro continuado del pH esofágico en $24 \mathrm{~h}$ es el método funcional más ójietivo para et estudio del reflujo gcstresscháçico, pues permite delerrinar parrones de exposición esolágico ol ácido y correlacionar diversos sintomas con el reflu $c$, siendo la pesquisa del reflujo cculto una de las prircipales indicaciones. La estandarización propuesta par lo Sociedad Eurcpeo de Gostroenlerología y Nutrición Pediärica [ESPGA.N] ha sido un gran aporte pora unitorrar le interpreación y comunicación de resuliados entre dferentes agrupaciones slínicas.
\end{abstract}

|Palabras clove: esćlogo, pH, registro conlinuado, reflujo gastrcesológico.|

\section{Continuous 24 hour esophageal pH monitoring in children}

Continuous 24-hour esophageal $\mathrm{pH}$ monitoring has been introduced ralher recently in Chile. II is considered one of the most objective functional method to study gastroesophageal reflux al present. It allows to delinate esophageal exposure to different patterns of acid reflux from gastric content and to correlate a variety of symptoms with gastroesophageal reflux. The confirmation of occult reflux is one of il's most important. The standards proposed by the European Society of Pediatrics Gastroenterology and Nutrition constitute a greal contribution to the uniformity of report and interpretation of results.

(Key words: esophagus, pH monitoring. gastroesophageal reflux.)

La medición continuada de $\mathrm{pH}$ esofágico en 24 horas es considerada el método funcional más objetivo y confiable para el estudio del reflujo gastroesofágico, pues detecta cambios de la acidez local que obedecen a los componentes regurgitados causantes de injuria con sensibilidad y especificidad superiores a $95 \%{ }^{1-4}$. Las características del método de sencillez y reproductibilidad la hacen ideal para identificar los episodios de reflujo ácido, medirlos objetivamente y determinar patrones de exposición de la nucosa esofágica al ácido.

El reflujo gastroesofágico es frecuente en la jnfancia y consiste en el paso retrógrado de contenido gástrico al esófago. Puede incluir saliva, alimentos o líquidos ingeridos, secreciones gástricas, así como pancreáticas o biliares que ha-

1. Servicio de Cirugía Hospital Luis Calvo Mackenna. yan refluido primariamente hacia el estomago 5 . Desde que fue descrito, en el año 1947, como entidad clínica en niños ${ }^{6}$, se ha observado un dramático incremento en el número de pacientes en que se diagnostica ${ }^{7}$. Concomitantemente se han desarrollado numerosos exámenes para medirlo y estudiar la fisiopatología de la afección.

\section{Fisiopatología}

El reflujo gastroesofágico es un fenómeno fisiológico que ocurre a lo largo de toda la vida, como consecuencia de la gradiente de presión existente entre el abdomen y el tórax ${ }^{8}$. Una barera antirreflujo modula el paso del contenido gástrico al esófago. Está constituida por componentes anatómicos (ángulo de His, crura diafragmática, esófago abdominal) funcionales 
(esfínter esofágico inferior), de cuya función depende en parte la cantidad del material refluido. El contenido gástrico también participa en este fenómeno en equilibrio con la barrera antes aludida, y está influido principalmente por el volumen del material ingerido, la capacidad de vaciamiento del estómago y la existencia y cantidad de reflujo duodenogástrico $0^{9,10}$.

Cuando se produce un desequilibrio entre estos factores el reflujo gastroesofágico se hace frecuente y puede causar enfermedad, la magnitud de la cual se asocia, en general, a la de la exposición del esófago al ácido. La enfermedad por reflujo gastroesofágico puede presentarse en los niños con variados síntomas de diferente intensidad, lo que hace necesario demostrar y medir objetivamente el fenómeno, para tratarlo de modo oportuno, previniendo complicaciones mavores.

En 1884 Reichman reprodujo dolor torácico en pacientes introduciéndoles una esponja con acido en esófago. Los primeros registros de reflujo gastroesofágico mediante determinación de $\mathrm{pH}$ esofágico fueron publicados el año $1958^{11}$. Posteriormente, en 1974, L. Johnson y T. DeMeester dan a conocer su experiencia sobre monitorización continua del $\mathrm{pH}$ en el esófago distal ${ }^{12}$. En los años 1977 y 1978 Hill y Jolley realizan las primeras determinaciones en niños $^{13}$. Durante los últimos 20 años se desarroIlaron equipos de fácil operación y menor coste que permitieron estandarizar los resultados para su interpretación, facilitado la amplia difusión y aceptación clínica de dichas determinaciones ${ }^{14.15}$.

\section{Procedimiento}

Para medir el $\mathrm{pH}$ de manera continuada durante 24 horas se requiere una unidad para el registro de la información, una de análisis, catéteres para la medición de $\mathrm{pH}$ y soluciones buffer.

La unidad de registro consiste en microcomputadores portátiles que permiten almacenar información análogo-digital durante un período prolongado con capacidad para varios canales de pH y presión y marcadores que permiten registrar eventos como alimentación, posición, dolor y otros síntomas.

La unidad de análisis está constituida por un programa especialmente diseñado que traspasa la información desde la de registro a un computador donde es analizada. El operador dispone. entonces, de un completo informe numérico y gráfico.

Existen dos tipos de catéteres para registro de $\mathrm{pH}$ en el esófago. Los de cristal permiten mediciones de aita resolución. Los de antimonio son los más empleados por ser baratos y reutilizables. Estos últimos miden la acidez utilizando el potencial de corrosión que se produce en la interfase protón/antimonio, requieren de un electrodo de referencia externo que se adhiere a la piel y tienen una vida útil garantizada de 150 horas. Estos catéteres son de diferentes largos y diámetros y pueden Ilevar incorporados varios canales de registro de $\mathrm{pH}^{16,17}$. Para la calibración del sistema a $\mathrm{pH} 7,01$ y 1,07 (a 25 grados Celcius) se emplean soluciones tampôn (buffers).

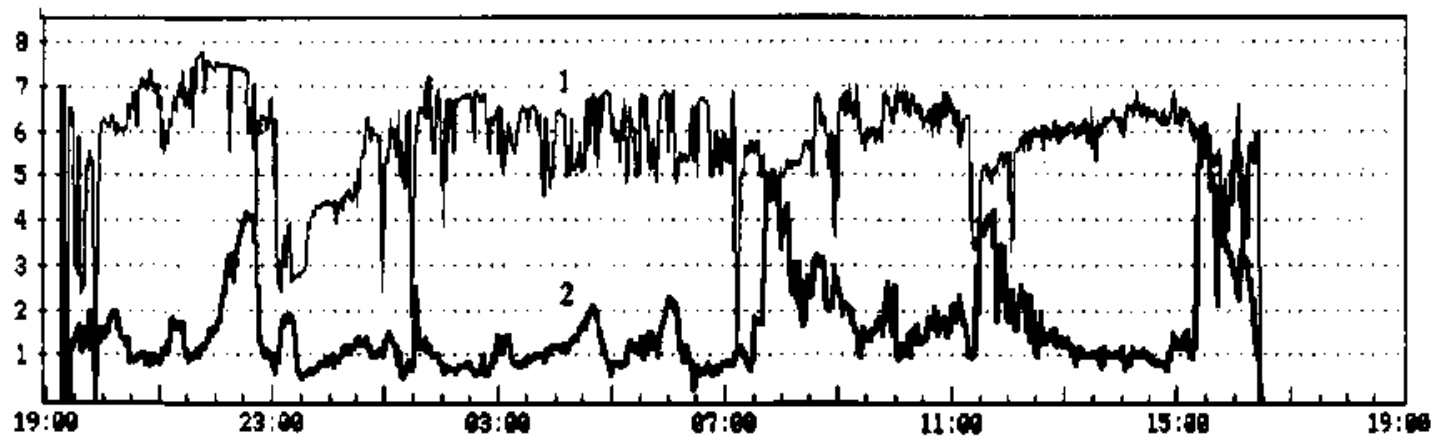

Figura: Registro continuo de $\mathrm{pH}$ esofágico y gástrico de 24 horas.

El trazado superior (1) corresponde al pH esofágico. Las caídas de la curva por debajo de pH 4 se interpretan como reflujo de ácido. El trazado inferior corresponde al registro sinnultáneo del pH gástrico (2) que fluctúa alrededor de pH 1 y 2 . Los ascensos del pH gástrico corresponden a la ingestión de alimentos. 
El método de registro, medición e interpretación de los resultados de $\mathrm{pH}$ esofágico ha sufrido numerosas modificaciones debidas al avance tecnológico y a la variedad de investigadores que trabajan en este campo $14.15,18$.

En junio del año 1991 el "Grupo de Trabajo de la Socicdad Europea de Nutrición y Gastroenterologia Pediatrica" (ESPGAN) recomendó la aplicación de un "Protocolo estándar del procedimicnto e interpretación de la monitoría prolongada del $\mathrm{pH}$ esofágico para el diagnóstico de reflujo gastrocsofágico"19 que hemos incorporado adaptándolo a la disponibilidad local de recursos con las siguientes recomendaciones:

Los medicamentos bloqueadores $\mathrm{H} 2$ deben ser incerrumpidos 3 o 4 dias antes del registro, los bloqueadores de la bomba de protones 15 días antes, los antiácidos el día del ingreso y los fármacos procinéticos 48 horas antes. Hemos optado por hospitalizar a los pacientes porque de este modo se obtiene información más confable y se evitan situaciones inconvenientes, descritas en otras experiencias, como retiro accidental de la sonda, desprendimiento de electrodos $y$ daños en los equipos. Se pide a los pacientes que ingresen en ayunas con la intención de que el estómago esté vaćó al momento de instalar la sonda.

Antes de instalar los catéteres de registro el sistema es calibrado a $\mathrm{pH} 1,07$ y 7,025 . Es recomendable verificar la calibración al tétmino de cada procedimiento. El electrodo se introduce por vía nasal, ubicando el extremo lejano del catéter en el tercio distal del esofago, para lo cual se usa como referencia el borde superior del cuerpo de la tercera vértebra por sobre el diafragma. La ubicación se confirma mediante radjografía de tórax ${ }^{10}$. En otros centros la posición de la punta del electrodo se establece con la fórmula de Strobel o con registros mano-métricos ${ }^{20,21}$. La fórmula de Strobel permite estimar la distancia entre la nariz y el esfínter esofágico inferior en centímetros y es igual a: 5 $+0,252$ - talla en $\mathrm{cm}$ y es válida en menores de uก ลกี้อ.

La actividad del paciente, en la medida que sea posible, debe scr normal, registrándose rigurosamente los cambios de posición y los horarios de sueño y vigilia, especialmente en pacienles con síntomas respiratorios, en los cuales los episodios nocturnos tienen influencia comprobada sobre la enfermedad pulmonar ${ }^{10}$.
La alimentación por lo general no se modifica. Idealmente debe ser lo más espaciada posible y en los casos en que es exclusivamente láctea sugerimos intervalos de al menos 4 horas entre una y otra ingestión, para lo cual nos basamos en estudios que muestran que la alcalinización gástrica posprandial puede durar hasta 200 minutos $y$ en nuestra experiencia, pues hemos observado que este fenomeno puede proJongarse aun más. Los alimentos calientes, las bebidas ácidas y gaseosas deben evitarse, pues alteran la sensibilidad de lps electrodos de antimonio y el pH esofagogástrico.

El tiempo de registro debe ser al menos de 18 horas, incluyendo un período nocturno y otro diurno?? comenzando no antes de 30 minutos después de la instalación del electrodo, debido a la hipersalivación que se produce ${ }^{19}$.

Las normas expuestas han permitido mejorar la sensibilidad y especificidad del método ${ }^{22-24}$. Los falsos positivos de reflujo gastroesofágico disminuyen al no suministrar alimentos ni bebidas acidas; lo mismo ocurre con los falsos negativos al identificar las situaciones en que existe una alcalinización gástrica prolongada (terapia ácido supresora, alimentación láctea continua o hipoacidez en nacidos prematuros y desnutridos), ya que en estos pacientes el paso de contenido gástrico al esófago no será valorado como expresión de reflujo gastroesofágico, puesto que por definición el método no considera reflujo si el pH del material registrado es mayor a 4 . Si se conoce el $\mathrm{pH}$ gástrico podremos detectar situaciones como las anteriormente descritas, razón por la que recomendamos medir simultáneamente el pH gástrico mediante sondas de 2 canales. Otras causas de registros o interpretación defectuosa son las sondas con exceso de uso o la impactación del cristal de antimonio en la mucosa.

Los programas de análisis disponibles entregan gran cantidad de información numérica $y$ gráfica 22-25. Para la evaluación del estudio se consideran el número de episodios ácidos, el de episodios de más de 5 minutos y la duración del episodio más largo; el porcentaje del tiempo de pH bajo 4: tiempo de la depuración o aclaramiento esofágico (clearance); área bajo la curva de $\mathrm{pH}$; indice oscilatorio e índice sintomático.

Se define como episodio ácido todo evento en el cual el pH esofágico es menor o igual a 4 y que dura más de 15 segundos $^{19}$. Esto se basa 
en que los seres humanos sienten dolor torácico cuando el pH esofágico es menor a 4 (Tutle 1961). Además la pepsina se activa bajo $\mathrm{pH} 4$. El número de episodios mayor de 5 minutos y la duración del episodio más largo expresan el mismo fenómeno y se correlacionan directamente con mayor incidencia de esofagitis péptica $^{22}$ 2. 2 . El porcentaje del tiempo de $\mathrm{pH}$ bajo 4 es considerado el parámetro más importante $y$ se emplea para discriminar entre sujetos normales y enfermos ${ }^{25}$. El aclaramiento o depuración esofágico ("clearance", traduce la capacidad del esófago para barrer el material refluido y restituir e] $\mathrm{pH}$ al existente antes del episodio de reflujo ${ }^{24}$. El área bajo la curva de $\mathrm{pH}$ permite evaluar el impacto del ácido refluente sobre la mucosa esofágica y posiblemente es un criterio de mayor especificidad y sensibilidad comparado con otros más antiguos, para predicción de csofagitis péptica ${ }^{26.27}$. El índice oscilatorio describe el porcentaje de tiempo en que el pH oscila entre 3,75 y $4,2528,29$. El índice sintomático describe la asociación entre el síntoma y los episodios de $\mathrm{pH}$ bajo 4 y se expresa en valores porcentuales ${ }^{30-32}$.

La combinación de todos estos criterios ha permitido determinar patrones de exposición al áciđo del esófago distal en niños sanos y enfermos que deben ser considerados valores de referencia, aplicables sólo si las poblaciones estudiadas son comparables ${ }^{22}$. Los patrones de exposición al ácido permiten correlacionar los resultados con determinadas afecciones clínicas, como por ejemplo de esofagitis ${ }^{33.34}$ y apnea. En el caso de los trastornos respiratorios, la descripción de un patrón específico, conocido como ZMD (promedio de duración de los episodios de reflujo ácido que ocurren durante el sueño después de al menos 2 horas de la última alimentación) ha sido de gran importancia, pues permite establecer una correlación entre los síntomas y el reflujo ${ }^{35-4]}$.

Siendo muchas las variables susceptibles de análisis y con la intención de agrupar sus valores en uno solo, se han diseñado distintas escalas de calificación, siendo las más difundidas las de T. DeMeester en adultos y de Boix Ochoa en niños ${ }^{\text {t2 }}$. Creemos que hay que ser cautelosos en la utilización de estas calificaciones, pues la interpretación de los resultados del examen debe realizarse analizando todas las variables por separado, especialmente en pacientes con síntomas específicos.

\section{Indicaciones para el estudio}

La Sociedad Norteamericana de Nutrición y Gastroenterología Pediátrica (NASPGN) en mayo de 1994 estableció las indicaciones para el estudio, señalando las circunstancias clínjcas en que el método tiene mayor utilidad y rendimiento como aporte al diagnóstico y evaluación terapéutica del paciente (tabla) ${ }^{1,43-49}$, lo que, en general, corresponde a aquellas en que se busca una relación de causalidad entre un síntoma $o$

\section{Tabla}

Indicaciones para la medición de $\mathrm{pH}$ esofágico

\begin{tabular}{|c|c|c|}
\hline $\begin{array}{c}\text { R gastroesofágico } \\
\text { oculto }\end{array}$ & $\begin{array}{l}\text { Correlacionar } \\
\text { con síntomas }\end{array}$ & Eraluación \\
\hline Bronconeumonía recurrente*. & Dolor torácico* & Tratamiento Inédico* \\
\hline S. bronquial obstr. recurrente* & Apnea & Trátamiento quifúrgico* \\
\hline Asma sef ractaria* & Llanto iniratable & vomitos \\
\hline Síntomas laríngeos* & Irtitabijidad & \\
\hline \multicolumn{3}{|l|}{ Falla sreciniento } \\
\hline Rechazo alimentos & & \\
\hline
\end{tabular}

R: refiujo: *: indicaciones en las que la medición de $\mathrm{pH}$ esofágico tiene mayor utilidad y rendiroiento. 
manifestación clínica y reflujo. En pacientes vomitadores el diagnóstico de reflujo gastroesofágico es clínico y la medición de $\mathrm{pH}$ esofagico no es necesaria para confirmarlo, siendo, en estos casos, relativa la del examen, ya que sólo entregará información complementaria sobre un reflujo ya identificado. La medición del $\mathrm{pH}$ en el esófago tiene gran aplicación en la evaluación de los resultados del tratamiento médico o quirúrgico del reflujo, especialmente si la cvolución del paciente no ha sido la esperada.

\section{Comentario}

La medición de ph esofágico continuada por 24 h es útil en la medida que se interprete sobre la base de los antecedentes clínicos y exámenes complementarios de cada paciente ${ }^{18.35 .50 .51}$. El registro continuado de $\mathrm{pH}$, por su especifīcidad, constituye el método funcional más objetivo para estudiar la acidificación esofágica ${ }^{25} .52$, sin embargo existe un porcentaje de falsos negativos de reflujo gastroesofágico ácido. Las recomendaciones elaboradas para su empleo, entre ellas las de ESPGAN que hemos delineado, tienden a aumentar la sensibilidad y reproductibilidad del método, así como medir con exactitud el $\mathrm{pH}$ esofágico durante un período prolongado de tiempo, conocer su ritmo circadiano y establecer patrones de exposicion al ácido del esófago que caractericen las diferentes formas de presentación del reflujo gastroesofágico.

El procedimiento no está diseñado para detectar reflujo biliar. pues no es capaz de distinguir este fenómeno de otras causas de alcalinización esofágica. Hasta ahora el único método válido para denostrar contenido biliar en esufago es el BILITEC 2000, que detecta pigmentos biliares por espectrofotometría ${ }^{53.55}$.

\section{Referencias}

1. Collerti $R$. Christie $D$, Orentein $S$ : Indications for pediatric esophageal pH monitoring. J Pediatr Gası. enterol Nutr 1995; 21 : 253-262.

2. Sondheimeir $J M$ : Continuous monitoring of distal esophageal $\mathrm{pH}$ : a diagtostic tesl for gastroesophageal reflux. J Pediatr 1980; 96: 804-807

3. Dalt L. Mazzolen S. Monsini G. Domzelli $F$. Sacchello $F$ : Diagnostic accuracy of $\mathrm{pH}$ monitoring in gastroesophageal reflux. Arch Dis Child 1980; 64: 14211426.
4. Jamieson JR. Stein HI, DeMeester T: Ambulatory 24-h esophageal pH monitoring: Normal values optimal tresholds, specificity, sensivitivy, reproducivility. Am J Gastroenterol 1992; 87: 1102-1111.

5. Vandenplas $Y$ : Reflux esophagitis in infant and children: A report from de working group on gastroesophageal reflux disease of de European Society of Paediatric Gastroenterology and Nutrition. J Pediatr Gastroenterol Nutr 1994: 18:413.422.

6. Neuhdirser E, Berenberg W: Cardioesophageal relaxation as a cause of vomiting in infant. Radiology 1947: 48:480.

7. Hebra A. Hoffinan $M$ : Gastroesophageal reflux in children. Pediatr Clın North Am 1993: 40: 1233-1251.

8. Pope $C h$ : Acid reflux disorders. N Engl J Med 1994; $331: 656-660$.

9. Holloway $R$, Orenstein S: Gastroesophageal disease in adult and children. Bailliere $\mathrm{Cl}$ Gastroenterol 1991; 5 : $337-370$.

14. Vaudenplas $Y$, Govierts $H$, Helven $R$. Sacre L: Gastroesophageal reflux, as measured by 24 -hours $\mathrm{pH}$ montoring, in 509 healthy infants screened for risk of sudden infont death syndrome. Pediatrics 1991; 88: 834.840 .

10. Orenriein 5 : Controversies in pediatric gasiroesophageal reflux.J Pediatr Gastroenterol Nutr 1992; 14: 338-348.

11. Tullle S, Grossman M: Detection of gastroesophageal reflux by simultaneous measurement of intraluminal pressure and $\mathrm{pH}$. Proceedings of Society for experimental biology and inedicine 1958: 98: 225-227.

12. Johnron $L$, DeWesfer $T$ : Twenty four hours $\mathrm{pH}$ monitoring of the distal esophagus. A quantitative measure of gastroesophageal reflux. Am J Gastroenterof $1974 ; 62: 325$.

13. Jofley S. Johnson D. Herbisf J: An assessment of gastroesophageal reflux in children by extended $\mathrm{pH}$ jonitoring of the distal esophagus. Surgery 1978: 84 : 16.

14. Johnson F, Joelssun B: Reproducibility of ambulatory esophageal pH monitoring. Gut 1988: 29: 886-889.

IS. Vundenplas $Y$, Heiven $R$. Goyvacrs $H$, Sacré L: Reproducibjlity of continuous 24 hours oesophageal $\mathrm{pH}$ monitoring in infants and children. Gut 1990;31: 374.377 .

16. Vatdenpias Y. Helven R, Goywatrs $H$ : Comparative study of glass antimiony electrodes for comlinuous oesophageal pH monitoring. Gut 1991: 32: 708-712.

17. Bennert $3: \mathrm{pH}$ ineasurement in the oesophagus. Bailliere's Clin Gastroenterol 1987; 1: 747-767.

18. Demeesier $T$. Technique indications and clinical use of 24 hours esophageal pH monitoring. I Thorac Cardioyase Surg 1980; 79:656-670.

19. Working Group of the European Society of Pediatric Gastronterology und Nutrition (ESPGAN): A standarized prolocol for methodology of esopbageal $\mathrm{pH}$ monitoring and interpretation of the data for the diagnosis of gastroesophageal reflux. J Pediatr Gastroenterol Nutr 1992; 14:467-471.

20. Srobel C. Byme $W$ : Correlation of esophngeal lengths in children whith height. J Pediatr 1979;94: 81-84.

21. Benllech $C$, Benatent $M$ : Localización del esfinter esof́agico inferior: Nueva fórmula. Cir Ped 1992: $5: 3$. 
22. Vondenplex $Y_{1}$ Loeb $H$ : The interpretation of esophageal monitoring da1a. Eur I Pediatr 1990; 149; 598 602 .

23. Vundenplar Y. Sacrê-Smiss L: Continuous 24-hours esophageal $\mathrm{pH}$ monitoring in 185 asyntomalic infants 0-15 months old. J Pediatr Gastroenterol Nutr 1987:6: 220.224

24 Koih A. Gitss R: Continuous 20-24 h esophageal pH monitoring in infancy. $J$ Petiatr Surg 1981; I6: I09113.

25. Brix Ochou J. Laftente JM. Gil-Vernef JM: Twenty four hour esophageal $\mathrm{pH}$ monitoring is gastroesophageal reflux. J Pedialr Surg 1980; I: 74-78.

26. Towar $J A$, itidierdo $M$. Eizaguire $d$ : The area under pH curve: a single figurc parameter representative of esophaseal acid exposure. J Pediarr Surg 1991: 26: $163-167$

27. V'tondenpiers Y, Franch-Gossens A, Sacre-Smits L: Area under pH: Advantage of a new parameter in the interpretation of esophageal $\mathrm{pH}$ monitoring data in iufarts. J Pediatr Gastroenterol Nutr 1989: 9: 34-39.

28. Witumabe $Y$. Catto-Smith A: The clinical significance of a prolonged stable $\mathrm{pH}$ around 4.0 in $24-\mathrm{h} \mathrm{pH}$ monitoring. J Pediatr Gastroenterol Nutr 1994; 19: 5057.

29. Vandenplas Y. Leputure R. Helven R: Dependibility of esophageal $\mathrm{pH}$ inoniroring data in infunts on cut-off limits: the oscilatory index. J Pediatr Gastroenterol Nutr 1990: 11:304-309

30. Johnsfon B, Lole $A$ : Symptom index as a marker of gastroesophageal reflux disease. Br J Surg 1992; 79: 1054-1055.

3[. Wiener G. Rtcitter J. Croper J, Wu W. Citstell D: The symptom index: A clinically important parameter of ambularory 24 hour esophageal $\mathrm{pH}$ monitoring. Am J Gastroenterol 1988; B.3: 358-36].

32. Breusulthof $R$, Smous $A$ : The syimptom index. A valuabie additional paraneter in 24 hour esophageal $\mathrm{JH}^{\mathrm{H}} \mathrm{r}^{2} \mathrm{cording}$. Am J Gastroenterol 1991: 86: I60-I64.

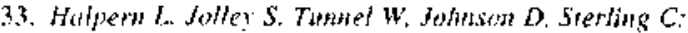
The mean duration of gastroesophageal reflux Juring sleep as an indicalor of respiratory symptoms from gastroesophageal reflux in children. J Pediatr Surg 1991: 26:686-690.

34. Vardenplats $\gamma$ : Reflux esophagitis in infants and ch1ldren: a report from the working group on gastrooesophageal reflux disease of the European Society of Pediatric Gastroemterology and Nutrition. I Pediat Gastroenterol Nutr 1994; 18: 41.3-422.

35. Jolley S. Herbst J. Johrson D. Mulak $M$, Book $L$. Esophageal pH monitoring duing sleep identifies children with respiratory symptoms from gastroesophageal retlux. 1981; 80; 1501-1506.

36. Jolley S. Hotpetw L. Tumel $W$, tohnon D. Sterting $C$ : The risk of sudden infant death from gasiroesophageal reflux. \& Pediatr Surg 159]; 26: 691-696.

37. Nelrytum L. Russe J. Olussmitn M. et al: Patterns of gastroesophageal reflux in patients whith apparent life threatening events J Pediatr Gastroenterol Nutr 1989; 8: $157-160$.

38. Gustafsnon P. Kjelintan N. Tibbling L: Bronchial asthma and acid reflux in to the distal and proximal oesophagus. Arch Dis Child 1990; 65: 1255-1258.
39. Orenstein $S$, Orenstein $D$ : Gastroesophageal reflux and respiratory disease in children. J Pediatr 1988; 1 12: $847-858$.

40. Eizagairre 1. Tovar J: Predicting preoperatively the outcome of respiratory symptoms of gastroesophingeal reflux. J Pediar Surg 1992: 27: 848-851.

4i. Euler $A$, Bywe $W$, Ament $M$ : Recurrent pulmonary disease in children: a complication of gastroesophageal teflux. Pediatrics 1979:63: 47-51.

42. Borx Ochoa $J$ : Radiation aspects of 24 hour $\mathrm{pH}$ monitoring. In DeMeester T. and Skinner D. Esophageal Disorders: Patophysiology and therapy New York: Raven Press 1985: 617

4.3. Andze $G$, Brand $M$ : Diagnosis and treatment of gastroesophageal reflux in 500 children whith respiralory symptoms: The value of $\mathrm{pH}$ monitoring. $\mathrm{J}$ Pediatr Surg 1991; 26: 295-300.

44. Pani M. Debass H. Pellegrini $C$ : Esophageal manometry and 24 hour $\mathrm{pH}$ monitoring in the diagnosis of pulmonary aspiration secundary to gastroesophageal reflux. Am J Surg 1992; 163: 401406.

45. Paterson W'. Abdallah $H$, Beck $I, D$ a Costa L: Ambulatory esophageal manometry, pH metry, and holter ECG monitoring in patients with atypical chest pain. Dig Dis Sci 1993: 38: 795-802.

46. Paterson W. Murat B: Combined ambulatory esophageal manometry and dual-probe pH merry in evaluation of patient with chronic unexplained cough. Dig Dis Sci 1994; 39: 1117-1125.

47. Tucci $F$, Resri $M$, Fomina $R$, Novembre $E$. Adami $C$, Vierucci A: Gastroesophageal reflux and bronchial asthma: prevalence and efect of cisapride therapy. J Pediatr Gastroenterol Nutr 1943; 17: 265-270.

48. Catw-Sinith A, Machida $H$. Butwer J. Gall $D$. Scott $R$ : The role of gastroesophagcal reflux in pediatric dysphagia. J Pediatr Surg 1991; 12: 159-165.

49. Herbst J. Minthon S, Book L: Gastroesophageal reflux causing respiratory distress and apnea in newborn infants, J Pediatr 1979: 95: 763-768.

50. Wesrat $S$, Dertx $H$, Tominiatu $\boldsymbol{d}$ : Symptomatic gastroesophageal reflux: Diagnosis with ultrasound. $J$ Pediatr Gastroenterol Nutr 1994: 19: 58-64.

51. Vandenpias $Y$. Derde M. Piepsz. A: Evaluation of reflux episodes during simuitaneous esophageal $\mathrm{pH}$ monitoring and gastroesophageal reflux. Scintigraphy in thildren. J Pediatr Gastroenterol Nutr 1952: 14: 256-260.

52. Schindbeck N. Heinvich C, Konig A, Dendorfer A: Optimal threshotds sensitivity, and specificity of longterm $\mathrm{pH}$ metry for the detection of gastroesophageal reftux disease. Gastroenterology 1987: 93: 85-90.

53. Becti P. Puchiani F, Baldini F, et al: Long-term ambulatory enterogastric reflux monitoring. Validalion of a new fiberoptic technique. Dig Dis Sci 1993: 38: $1237 \cdot 1306$

54. Calduell $M$. Byrne $P$, Brazil $N$. et al: An ambulatory bile reflux monitoring system: an in vitro appraisal. Physiol Meas 1994: 15: 57-67.

55. Katser W. Burdiles P. Jreland A, ef ad: Does duodenal juice reflux in 10 the esophagus on patients whith complicated GERD?. Evaluation of fiber optie sensor for bilirubin. Am J Surg 1995; 169: 98-104. 